\title{
Approximation by One and Two Variables of the Bernstein- Schurer-Type Operators and Associated GBS Operators on Symmetrical Mobile Interval
}

\author{
Reşat Aslan $(\mathbb{D})$ and Aydın İzgi \\ Department of Mathematics, Faculty of Sciences and Arts, Harran University, 63100 Haliliye, Şanliurfa, Turkey \\ Correspondence should be addressed to Reşat Aslan; resat63@hotmail.com
}

Received 5 March 2021; Revised 31 March 2021; Accepted 10 April 2021; Published 4 May 2021

Academic Editor: Tuncer Acar

Copyright (C) 2021 Reşat Aslan and Aydın İzgi. This is an open access article distributed under the Creative Commons Attribution License, which permits unrestricted use, distribution, and reproduction in any medium, provided the original work is properly cited.

\begin{abstract}
In this article, we purpose to study some approximation properties of the one and two variables of the Bernstein-Schurer-type operators and associated GBS (Generalized Boolean Sum) operators on a symmetrical mobile interval. Firstly, we define the univariate Bernstein-Schurer-type operators and obtain some preliminary results such as moments, central moments, in connection with a modulus of continuity, the degree of convergence, and Korovkin-type approximation theorem. Also, we derive the Voronovskaya-type asymptotic theorem. Further, we construct the bivariate of this newly defined operator, discuss the order of convergence with regard to Peetre's K-functional, and obtain the Voronovskaya-type asymptotic theorem. In addition, we consider the associated GBS-type operators and estimate the order of approximation with the aid of mixed modulus of smoothness. Finally, with the help of the Maple software, we present the comparisons of the convergence of the bivariate Bernstein-Schurer-type and associated GBS operators to certain functions with some graphical illustrations and error estimation tables.
\end{abstract}

\section{Introduction}

In [1], Bernstein suggested his polynomials that still inspire many studies today as follows:

$$
B_{r}(\mu ; x)=\sum_{j=0}^{r}\left(\begin{array}{l}
r \\
j
\end{array}\right) x^{j}(1-x)^{r-j} \mu\left(\frac{j}{r}\right), \quad x \in[0,1],
$$

for any $r \in \mathbb{N}$ and any $\mu \in C[0,1]$.

In 1962, the operators $S_{r}(\mu ; x): C[0, p+1] \longrightarrow C[0,1]$, which are called Bernstein-Schurer, are proposed by Schurer [2] as follows:

$$
S_{r}(\mu ; x)=\sum_{j=0}^{r+p}\left(\begin{array}{c}
r+p \\
j
\end{array}\right) x^{j}(1-x)^{r+p-j} \mu\left(\frac{j}{r}\right), \quad x \in[0,1],
$$

for any $r \in \mathbb{N}$ and $\mu \in C[0, p+1]$.
Very recently, many modifications and generalizations of the Bernstein or Bernstein-Schurer operators for univariate and bivariate cases are discussed by many authors. For instance, Acar et al. [3] established local and global approximation results in terms of modulus of continuity for a new type of the Bernstein-Durrmeyer operators on mobile interval. Izgi [4] presented a new type of the Bernstein polynomials and studied several approximation results of the univariate and bivariate of these operators. For the parameter $\alpha \in \mathbb{R}$, Chen et al. [5] defined a new generalization of the Bernstein operator and derived the order of convergence and Voronovskaya-type asymptotic relation for the $\alpha-$ Bernstein operator. Kajla and Acar [6] constructed a new kind of the $\alpha$-Bernstein operator and studied a uniform convergence estimate, some direct results involving the asymptotic theorems for these operators. Acar et al. [7] introduced the Kantorovich modifications of the $(p, q)$ - Bernstein operators for bivariate functions using a new $(p, q)$-integral and obtained the uniform convergence and rate of approximation 
in terms of modulus of continuity for these operators. Further, for $\lambda \in[-1,1]$, Cai [8] introduced the Bézier version of the Kantorovich-type $\lambda$-Bernstein polynomials and gained the global and direct approximation theorems. Acar and Kajla [9] introduced an extension of the bivariate generalized Bernstein operators with nonnegative real parameters and studied the degree of approximation with regard to Peetre's $K$-functional and Lipschitz-type functions. Bărbosu [10] demonstrated the uniform convergence and estimated the degree of approximation of the bivariate of the Bernstein-Schurer operators. Căbulea [11] considered the generalizations of the Kantorovich and Durrmeyer type of the Bernstein-Schurer operators and evaluated in connection with the modulus of continuity the order of approximation of these operators. Also, for some recent works, we can refer the readers to ([12-23]).

By the motivation of the all the above-mentioned works, we define the univariate Bernstein-Schurer-type operators on a symmetrical mobile interval. Let the intervals be $D_{r}=[-r /$ $(r+1)-p, r /(r+1)+p], I=[-1,1]$, and $C\left(D_{r}\right)$ be the set of all continuous and bounded functions on $D_{r}$. For a function $\mu \in C\left(D_{r}\right)$ and $x \in D_{r}$, the univariate Bernstein-Schurer operators $F_{r}: C\left(D_{r}\right) \longrightarrow C(I)$ are defined as

$$
F_{r}(\mu ; x)=\left(\frac{r+1}{2 r}\right) \sum_{j=0}^{r+p} \varphi_{r, j}^{p+p}(x) \mu\left(\frac{2 j-r}{r+1}\right)
$$

where $\in \mathbb{N}, p \in \mathbb{N}_{0}=\mathbb{N} \cup\{0\}, \varphi_{r, j}^{p}(x)=\left(\begin{array}{c}r+p \\ j\end{array}\right)(r /(r+1)+$ $x)^{j}(r /(r+1)-x)^{r+p-j}$.

The goal of the present work is to obtain some approximation features of the operators given by (3). We show the uniform convergence, estimate the degree of convergence with the help of modulus of continuity, and prove the Voronovskaya-type asymptotic theorem for the (3) operators. Next, we define the bivariate of (3) operators, compute the order of convergence by using Peetre's $K$-functional, and derive the Voronovskaya-type asymptotic theorem for the bivariate case. Further, we construct the associated GBS type of bivariate operators and estimate their degree of convergence in terms of mixed modulus of smoothness. Finally, by the help of the Maple software, we give comparisons of the convergence of bivariate of (3) operators and related GBS operators to the certain functions with some graphics and error estimation tables.

\section{Main Results}

Lemma 1. Let the operators $F_{r}(\mu ; x)$ be defined by (3). Then, for all $x \in D_{r}$, the following moments verify

$$
\begin{aligned}
& F_{r}(1 ; x)=1, \\
& F_{r}(t ; x)=x+\frac{p((r+1) x+r)}{r(r+1)},
\end{aligned}
$$

$$
F_{r}\left(t^{2} ; x\right)=x^{2}+\frac{\left(p^{2}+2 p r-p-r\right)}{r^{2}} x^{2}+\frac{2 p(r+p)}{r(r+1)} x+\frac{p^{2}+p+r}{(r+1)^{2}},
$$

$$
\begin{aligned}
F_{r}\left(t^{3} ; x\right) & \\
= & x^{3}+\frac{\left(p^{3}+3 p r^{2}+3 p^{2} r-3 p^{2}-3 r^{2}-3 p r+2 p+2 r\right)}{r^{3}} x^{3} \\
& +\frac{3 p(r+p)(r+p-1)}{r^{2}(r+1)} x^{2}+\frac{(r+p)\left(3 p^{2}+3 p+3 r-2\right)}{r(r+1)^{2}} x \\
& +\frac{p\left(p^{2}+3 p+3 r\right)}{(r+1)^{3}},
\end{aligned}
$$

$$
\begin{aligned}
F_{r}\left(t^{4} ; x\right) & \\
= & x^{4}+\left(\frac{p^{4}+4 p^{3} r+6 p^{2} r^{2}+4 p r^{3}-6 p^{3}-6 r^{3}-18 p r^{2}-18 p^{2} r}{r^{4}}\right. \\
& \left.+\frac{11 p^{2}+22 p r+11 r^{2}-6 p-6 r}{r^{4}} x^{4}\right) \\
& +\frac{4(p-1)(r+p)(r+p-1)(r+p-2)}{r^{3}(r+1)} x^{3} \\
& +\frac{2(r+p)(r+p-1)\left(3 p^{2}-3 p-3 r+8\right)}{r^{2}(r+1)^{2}} x^{2} \\
& +\frac{4(r+p)\left(p^{3}-3 p r-3 r^{2}+7 p+9 r-6\right)}{r(r+1)^{3}} x \\
& +\frac{p^{4}+2 p^{3}-6 p^{2} r-12 p r^{2}-4 r^{3}+15 p^{2}+30 p r+15 r^{2}-10 p-10 r}{(r+1)^{4}} .
\end{aligned}
$$

Proof. From (3), it becomes

$$
\begin{aligned}
F_{r}(1 ; x)= & \left(\frac{r+1}{2 r}\right)^{r+p} \sum_{j=0}^{r+p}\left(\begin{array}{c}
r+p \\
j
\end{array}\right)\left(\frac{r}{r+1}+x\right)^{j}\left(\frac{r}{r+1}-x\right)^{r+p-j} \\
= & \left(\frac{r+1}{2 r}\right)^{r+p}\left(\frac{2 r}{r+1}\right)^{r+p}=1, \\
F_{r}(t ; x)= & \left(\frac{r+1}{2 r}\right)^{r+p} \sum_{j=0}^{r+p}\left(\begin{array}{c}
r+p \\
j
\end{array}\right)\left(\frac{r}{r+1}+x\right)^{j} \\
& \cdot\left(\frac{r}{r+1}-x\right)^{r+p-j}\left(\frac{2 j-r}{r+1}\right) \\
= & \left.\left(\frac{r+1}{2 r}\right)^{r+p} \frac{2(r+p)^{r+p-1}}{r+1} \sum_{j=0}^{r+p-1}\right)^{j} \\
& \cdot\left(\frac{r}{r+1}+x\right)^{j+1}\left(\frac{r}{r+1}-x\right)^{r+p-j-1}-\frac{r}{r+1} \\
& \cdot\left(\frac{r+1}{2 r}\right)^{r+p} \sum_{j=0}^{r+p}\left(\begin{array}{c}
r+p \\
j
\end{array}\right)\left(\frac{r}{r+1}+x\right)^{j}\left(\frac{r}{r+1}-x\right)^{r+p-j} \\
= & \frac{r+1}{2 r} \frac{2(r+p)}{r+1}\left(\frac{r}{r+1}+x\right)-\frac{r}{r+1}=x+\frac{p((r+1) x+r)}{r(r+1)},
\end{aligned}
$$




$$
\begin{aligned}
& F_{r}\left(t^{2} ; x\right)=\left(\frac{r+1}{2 r}\right) \sum_{j=0}^{r+p}\left(\begin{array}{c}
r+p \\
j
\end{array}\right)\left(\frac{r}{r+1}+x\right)^{j} \\
& \cdot\left(\frac{r}{r+1}-x\right)^{r+p-j}\left(\frac{2 j-r}{r+1}\right)^{2} \\
& =\left(\frac{r+1}{2 r}\right)^{r+p} \frac{4(r+p)(r+p-1)}{(r+1)^{2}} \sum_{j=0}^{r+p-2}\left(\begin{array}{c}
r+p-2 \\
j
\end{array}\right) \\
& \cdot\left(\frac{r}{r+1}+x\right)^{j+2}\left(\frac{r}{r+1}-x\right)^{r+p-j-2} \\
& +\left(\frac{r+1}{2 r}\right)^{r+p} \frac{4(r+p)}{(r+1)^{2}} \sum_{j=0}^{r+p-1}\left(\begin{array}{c}
r+p-1 \\
j
\end{array}\right) \\
& \cdot\left(\frac{r}{r+1}+x\right)^{j+1}\left(\frac{r}{r+1}-x\right)^{r+p-j-1}-\left(\frac{r+1}{2 r}\right)^{r+p} \\
& \frac{4 r(r+p)}{(r+1)^{2}} \sum_{j=0}^{r+p-1}\left(\begin{array}{c}
r+p-1 \\
j
\end{array}\right)\left(\frac{r}{r+1}+x\right)^{j+1} \\
& \cdot\left(\frac{r}{r+1}-x\right)^{r+p-j-1}+\left(\frac{r+1}{2 r}\right)^{r+p}\left(\frac{r}{r+1}\right)^{2} \sum_{j=0}^{r+p}\left(\begin{array}{c}
r+p \\
j
\end{array}\right) \\
& \cdot\left(\frac{r}{r+1}+x\right)^{j}\left(\frac{r}{r+1}-x\right)^{r+p-j} \\
& =\left(\frac{r+1}{2 r}\right)^{2} \frac{4(r+p)(r+p-1)}{(r+1)^{2}}\left(\frac{r}{r+1}+x\right)^{2} \\
& +\left(\frac{r+1}{2 r}\right) \frac{4(r+p)}{(r+1)^{2}}\left(\frac{r}{r+1}+x\right)-\left(\frac{r+1}{2 r}\right) \frac{4 r(r+p)}{(r+1)^{2}} \\
& \cdot\left(\frac{r}{r+1}+x\right)+\left(\frac{r}{r+1}\right)^{2} \\
& =x^{2}+\frac{\left(p^{2}+2 p r-p-r\right)}{r^{2}} x^{2}+\frac{2 p(r+p)}{r(r+1)} x+\frac{p^{2}+p+r}{(r+1)^{2}} \text {. }
\end{aligned}
$$

The last two identities can be obtained by applying similar methods; hence, we have omitted the details.

Lemma 2. For all $x \in D_{r}$, we obtain the following central moments:

$$
\begin{gathered}
F_{r}(t-x ; x)=\frac{p((r+1) x+r)}{r(r+1)}, \\
F_{r}\left((t-x)^{2} ; x\right)=\frac{\left(p^{2}-p-r\right)}{r^{2}} x^{2}+\frac{2 p^{2}}{r(r+1)} x+\frac{p^{2}+p+r}{(r+1)^{2}}, \\
F_{r}\left((t-x)^{4} ; x\right) \\
=\frac{\left(p^{4}-6 p^{3}-6 p^{2} r-12 p r^{2}+11 p^{2}+14 p r+3 r^{2}-6 p-6 r\right)}{r^{4}} x^{4} \\
+\frac{4\left(p^{4}-4 p^{3}-6 p^{2} r-3 p r^{2}+5 p^{2}+8 p r+3 r^{2}-2 p-2 r\right)}{r^{3}(r+1)} x^{3} \\
+\frac{2\left(3 p^{4}-6 p^{3}-18 p^{2}-18 p r^{2}+11 p^{2}+26 p r+15 r^{2}-8 p-8 r\right)}{r^{2}(r+1)^{2}} x^{2} \\
+\frac{4\left(p^{4}-6 p^{2} r-9 p r^{2}+7 p^{2}+16 p r+9 r^{2}-6 p-6 r\right)}{r(r+1)^{3}} x \\
+\frac{p^{4}+2 p^{3}-6 p^{2} r-12 p r^{2}+15 p^{2}+30 p r+15 r^{2}-10 p-10 r}{(r+1)^{4}} .
\end{gathered}
$$

Proof. The proof of this lemma can be directly obtained by using the linearity of (3) operators and as a consequence of Lemma 1.

Corollary 3. For all $x \in D_{r}$, the following identities hold:

$$
\begin{aligned}
\lim _{r \longrightarrow \infty} r\left(F_{r}(t-x ; x)\right) & =p(x+1), \\
\lim _{r \longrightarrow \infty} r\left(F_{r}\left((t-x)^{2} ; x\right)\right) & =1-x^{2}, \\
\lim _{r \longrightarrow \infty} r^{2}\left(F_{r}\left((t-x)^{4} ; x\right)\right) & =3\left(1-3 x^{2}\right)\left(x^{2}+1\right) .
\end{aligned}
$$

In the next theorem we show the uniform convergence of (3) operators. As it is known, the space $C\left(D_{r}\right)$ denotes the real-valued continuous functions on $D_{r}$, and it is equipped with the norm for a function $\mu$ as follows:

$$
\|\mu\|_{C\left(D_{r}\right)}=\sup _{x \in D_{r}}|\mu(x)|
$$

Theorem 4. Let the operators $F_{r}(\mu ; x)$ be given by (3). Then, for all $x \in D_{r}, F_{r}(\mu ; x)$ converges to $\mu$ uniformly on $D_{r}$.

Proof. From (4), it is obvious that

$$
\lim _{r \longrightarrow \infty}\left\|F_{r}(1 ; .)-1\right\|_{C\left(D_{r}\right)}=0 .
$$

By (5), we arrive

$$
\begin{aligned}
\lim _{r \longrightarrow \infty}\left\|F_{r}(t ; .)-x\right\|_{C\left(D_{r}\right)} & =\lim _{r \longrightarrow \infty} \max _{x \in D}\left|p x \frac{(2 r+1)}{r(r+1)}\right| \\
& <\lim _{r \longrightarrow \infty}\left|\frac{p(p r+p+2 r)}{r(r+1)}\right|=0 .
\end{aligned}
$$

Similarly, using (6), then

$$
\begin{aligned}
& \lim _{r \rightarrow \infty}\left\|F_{r}\left(t^{2} ; .\right)-x^{2}\right\|_{C\left(D_{r}\right)} \\
& \quad=\lim _{r \longrightarrow \infty} \max _{x \in D_{r}}\left|\frac{\left(p^{2}-p-r\right)}{r^{2}} x^{2}+\frac{2 p^{2}}{r(r+1)} x+\frac{p^{2}+p+r}{(r+1)^{2}}\right| \\
& \quad<\lim _{r \rightarrow \infty}\left|\frac{p^{2}-3 p+r+4}{(r+1)^{2}}\right|=0 .
\end{aligned}
$$

Hence, according to the Korovkin theorem [24], the (3) operators converge uniformly to $\mu$ on $D_{r}$.

Further, we will obtain the degree of approximation of (3) operators. Let the modulus of continuity for a function $\mu \in$ $C[a, b]$ be given by

$$
\omega(\mu, \delta)=\sup _{\substack{t, y \in[a, b] \\|t-y| \leq \delta}}|\mu(t)-\mu(y)| .
$$

Since $\delta>0, \omega(\mu, \delta)$ has some useful properties which can be found in [25]. 
Theorem 5. Let $\mu \in C\left(D_{r}\right)$. Then, for every $x \in D_{r}$, the following inequality is verified:

$$
\left|F_{r}(\mu ; x)-\mu(x)\right| \leq 2 \omega\left(\mu ; \gamma_{r}(x)\right)
$$

where $\gamma_{r}(x):=\sqrt{F_{r}\left((t-x)^{2} ; x\right)}$.

Proof. Taking into account the following common property of the modulus of continuity:

$$
|\mu(t)-\mu(x)| \leq\left(1+\frac{|t-x|}{\delta}\right) \omega(\mu ; \delta),
$$

by the linearity of the operator (3), then

$$
\left|F_{r}(\mu ; x)-\mu(x)\right| \leq\left(1+\frac{1}{\delta} F_{r}(|t-x| ; x)\right) \omega(\mu ; \delta) .
$$

Utilizing the Cauchy-Schwarz inequality yields

$$
\begin{aligned}
\left|F_{r}(\mu ; x)-\mu(x)\right| & \leq\left(1+\frac{1}{\delta} \sqrt{F_{r}\left((t-x)^{2} ; x\right)}\right) \omega(\mu ; \delta) \\
& \leq\left(1+\frac{\gamma_{r}(x)}{\delta}\right) \omega(\mu ; \delta) .
\end{aligned}
$$

If we take $\delta=\gamma_{r}(x):=\sqrt{F_{r}\left((t-x)^{2} ; x\right)}$, thus Theorem 5 is proven.

Theorem 6. Let the operators $F_{r}(\mu ; x)$ be given by (3). Then, for any $\mu \in C\left(D_{r}\right)$ such that $\mu^{\prime}, \mu^{\prime \prime} \in C\left(D_{r}\right)$, the following identity holds:

$$
\lim _{r \longrightarrow \infty} r\left(F_{r}(\mu ; x)-\mu(x)\right)=p(x+1) \mu^{\prime}(x)+\frac{1}{2}\left(1-x^{2}\right) \mu^{\prime \prime}(x),
$$

uniformly on $D_{r}$.

Proof. Suppose that $\mu, \mu^{\prime}, \mu^{\prime \prime} \in C\left(D_{r}\right)$ and $x \in D_{r}$ are fixed. By the Taylor formula, hence

$\mu(t)=\mu(x)+\mu^{\prime}(x)(t-x)+\frac{1}{2} \mu^{\prime \prime}(x)(t-x)^{2}+\xi(t ; x)(t-x)^{2}$,

where $\xi(t ; x)$ is a form of Peano of the rest term, and since $\xi(. ; x) \in C\left(D_{r}\right), \lim _{t \rightarrow x} \xi(t ; x)=0$.

Operating $F_{r}(. ; x)$ to $(22)$, then

$$
\begin{aligned}
F_{r}(\mu ; x)-\mu(x)= & \mu^{\prime}(x) F_{r}(t-x ; x)+\frac{1}{2} \mu^{\prime \prime}(x) F_{r}\left((t-x)^{2} ; x\right) \\
& +F_{r}\left(\xi(t ; x)(t-x)^{2} ; x\right) .
\end{aligned}
$$

From Lemma 2, it becomes

$$
\begin{aligned}
\lim _{r \rightarrow \infty} r\left(F_{r}(\mu ; x)-\mu(x)\right)= & p(x+1) \mu^{\prime}(x)+\frac{1}{2}\left(1-x^{2}\right) \mu^{\prime \prime}(x) \\
& +\lim _{r \rightarrow \infty} r\left(F_{r}\left(\xi(t ; x)(t-x)^{2} ; x\right)\right) .
\end{aligned}
$$

Applying the Cauchy-Schwarz inequality, one has

$r\left(F_{r}\left(\xi(t ; x)(t-x)^{2} ; x\right)\right) \leq \sqrt{F_{r}\left(\xi^{2}(t ; x) ; x\right)} \sqrt{r^{2} F_{r}\left((t-x)^{4} ; x\right)}$.

Owing to $\xi(t ; x) \in C\left(D_{r}\right), \lim _{t \longrightarrow x} \xi(t ; x)=0$, thus

$$
\lim _{r \longrightarrow \infty} F_{r}\left(\xi^{2}(t ; x) ; x\right)=0,
$$

uniformly on $D_{r}$ with Theorem 4 .

Combining (25) and (26) and by Lemma 2, we get

$$
\lim _{r \longrightarrow \infty} r\left(F_{r}\left(\xi(t ; x)(t-x)^{2} ; x\right)\right)=0 .
$$

Hence,

$\lim _{r \longrightarrow \infty} r\left(F_{r}(\mu ; x)-\mu(x)\right)=p(x+1) \mu^{\prime}(x)+\frac{1}{2}\left(1-x^{2}\right) \mu^{\prime \prime}(x)$,

which gives the proof.

\section{Construction of the Bivariate Bernstein- Schurer-Type Operators}

Let the intervals be $D_{r_{1}, r_{2}}=\left[\left(-r_{1} / r_{1}+1\right)-p_{1},\left(r_{1} / r_{1}+1\right)+p_{1}\right]$ $\times\left[\left(-r_{2} / r_{2}+1\right)-p_{2},\left(r_{2} / r_{2}+1\right)+p_{2}\right], I^{2}=I \times I=[-1,1] \times[-$ $1,1]$, and by $C\left(D_{r_{1}, r_{2}}\right)$, we denote the set of all real-valued continuous functions on $D_{r_{1}, r_{2}}$, and it is equipped with the norm $\|\mu\|_{C\left(D_{r_{1}, r_{2}}\right)}=\sup _{(x, y) \in D_{r_{1}, r_{2}}}|\mu(x, y)|$.

We define the bivariate $F_{r_{1}, r_{2}}: C\left(D_{r_{1}, r_{2}}\right) \longrightarrow C\left(I^{2}\right)$ of operators given by (3) as follows:

$$
\begin{aligned}
F_{r_{1}, r_{2}}(\mu ; x, y)= & \left(\frac{r_{1}+1}{2 r_{1}}\right)^{r_{1}+p_{1}}\left(\frac{r_{2}+1}{2 r_{2}}\right)^{r_{2}+p_{2}} \sum_{j_{1}=0}^{r_{1}+p_{1}} \sum_{j_{2}=0}^{r_{2}+p_{2}} \varphi_{r_{1}, r_{2}, j_{1}, j_{2}}^{p_{1}, p_{2}} \\
& \cdot(x, y) \mu\left(\frac{2 j_{1}-r_{1}}{r_{1}+1}, \frac{2 j_{2}-r_{2}}{r_{2}+1}\right),
\end{aligned}
$$

where $\varphi_{r_{1}, r_{2}, j_{1}, j_{2}}^{p_{1}, p_{2}}(x, y)=\left(\begin{array}{c}r_{1}+p_{1} \\ j_{1}\end{array}\right)\left(\begin{array}{c}r_{2}+p_{2} \\ j_{2}\end{array}\right)\left(r_{1} /\left(r_{1}+1\right)+\right.$ $x)^{j_{1}}\left(r_{2} /\left(r_{2}+1\right)+y\right)^{j_{2}}\left(r_{1} /\left(r_{1}+1\right)-x\right)^{r_{1}+p_{1}-j_{1}} \quad\left(r_{2} /\left(r_{2}+1\right)-\right.$ $y)^{r_{2}+p_{2}-j_{2}},\left(r_{1}, r_{2}\right) \in \mathbb{N} \times \mathbb{N},\left(p_{1}, p_{2}\right) \in \mathbb{N}_{0} \times \mathbb{N}_{0}$, and $(x, y) \in$ $D_{r_{1}, r_{2}}$. 
It can be seen that the operators given by (29) are positive and linear.

Lemma 7. Let $e_{a_{1}, a_{2}}(x, y)=x^{a_{1}} y^{a_{2}},(x, y) \in D_{r_{1}, r_{2}},\left(a_{1}, a_{2}\right) \in \mathbb{N}_{0}$ $\times \mathbb{N}_{0}$ with $a_{1}+a_{2} \leq 4$, be the bivariate test functions. Then, one has

$$
\begin{aligned}
& F_{r_{1}, r_{2}}\left(e_{0,0} ; x, y\right)=1 \text {, } \\
& F_{r_{1}, r_{2}}\left(e_{1,0} ; x, y\right)=x+\frac{p_{1}\left(\left(r_{1}+1\right) x+r_{1}\right)}{r_{1}\left(r_{1}+1\right)}, \\
& F_{r_{1}, r_{2}}\left(e_{0,1} ; x, y\right)=y+\frac{p_{2}\left(\left(r_{2}+1\right) y+r_{2}\right)}{r_{2}\left(r_{2}+1\right)}, \\
& F_{r_{1}, r_{2}}\left(e_{2,0} ; x, y\right) \\
& =x^{2}+\frac{\left(p_{1}^{2}+2 p_{1} r_{1}-p_{1}-r_{1}\right)}{r_{1}^{2}} x^{2}+\frac{2 p_{1}\left(r_{1}+p_{1}\right)}{r_{1}\left(r_{1}+1\right)} x+\frac{p_{1}^{2}+p_{1}+r_{1}}{\left(r_{1}+1\right)^{2}}, \\
& F_{r_{1}, r_{2}}\left(e_{0,2} ; x, y\right) \\
& =y^{2}+\frac{\left(p_{2}^{2}+2 p_{2} r_{2}-p_{2}-r_{2}\right)}{r_{2}^{2}} y^{2}+\frac{2 p_{2}\left(r_{2}+p_{2}\right)}{r_{2}\left(r_{2}+1\right)} y+\frac{p_{2}^{2}+p_{2}+r_{2}}{\left(r_{2}+1\right)^{2}}, \\
& F_{r_{1}, r_{2}}\left(e_{3,0} ; x, y\right) \\
& =x^{3}+\frac{\left(p_{1}^{3}+3 p_{1} r_{1}^{2}+3 p_{1}^{2} r_{1}-3 p_{1}^{2}-3 r_{1}^{2}-3 p_{1} r_{1}+2 p_{1}+2 r_{1}\right)}{r_{1}^{3}} x^{3} \\
& +\frac{3 p_{1}\left(r_{1}+p_{1}\right)\left(r_{1}+p_{1}-1\right)}{r_{1}^{2}\left(r_{1}+1\right)} x^{2}+\frac{\left(r_{1}+p_{1}\right)\left(3 p_{1}^{2}+3 p_{1}+3 r_{1}-2\right)}{r_{1}\left(r_{1}+1\right)^{2}} x \\
& +\frac{p_{1}\left(p_{1}^{2}+3 p_{1}+3 r_{1}\right)}{\left(r_{1}+1\right)^{3}} \\
& F_{r_{1}, r_{2}}\left(e_{0,3} ; x, y\right) \\
& =y^{3}+\frac{\left(p_{2}^{3}+3 p_{2} r_{2}^{2}+3 p_{2}^{2} r_{2}-3 p_{2}^{2}-3 r_{2}^{2}-3 p_{2} r_{2}+2 p_{2}+2 r_{2}\right)}{r_{2}^{3}} y^{3} \\
& +\frac{3 p_{2}\left(r_{2}+p_{2}\right)\left(r_{2}+p_{2}-1\right)}{r_{2}^{2}\left(r_{2}+1\right)} y^{2}+\frac{\left(r_{2}+p_{2}\right)\left(3 p_{2}^{2}+3 p_{2}+3 r_{2}-2\right)}{r_{2}\left(r_{2}+1\right)^{2}} y \\
& +\frac{p_{2}\left(p_{2}^{2}+3 p_{2}+3 r_{2}\right)}{\left(r_{2}+1\right)^{3}} \\
& F_{r_{1}, r_{2}}\left(e_{4,0} ; x, y\right) \\
& =x^{4}+\left(\frac{p_{1}^{4}+4 p_{1}^{3} r_{1}+6 p_{1}^{2} r_{1}^{2}+4 p_{1} r_{1}^{3}-6 p_{1}^{3}-6 r_{1}^{3}-18 p_{1} r_{1}^{2}-18 p_{1}^{2} r_{1}}{r_{1}^{4}}\right. \\
& \left.+\frac{11 p_{1}^{2}+22 p_{1} r_{1}+11 r_{1}^{2}-6 p_{1}-6 r_{1}}{r_{1}^{4}}\right) x^{4} \\
& +\frac{4\left(p_{1}-1\right)\left(r_{1}+p_{1}\right)\left(r_{1}+p_{1}-1\right)\left(r_{1}+p_{1}-2\right)}{r_{1}^{3}\left(r_{1}+1\right)} x^{3} \\
& +\frac{2\left(r_{1}+p_{1}\right)\left(r_{1}+p_{1}-1\right)\left(3 p_{1}^{2}-3 p_{1}-3 r_{1}+8\right)}{r_{1}^{2}\left(r_{1}+1\right)^{2}} x^{2} \\
& +\frac{4\left(r_{1}+p_{1}\right)\left(p_{1}^{3}-3 p_{1} r_{1}-3 r_{1}^{2}+7 p_{1}+9 r_{1}-6\right)}{r_{1}\left(r_{1}+1\right)^{3}} x \\
& +\frac{p_{1}^{4}+2 p_{1}^{3}-6 p_{1}^{2} r_{1}-12 p_{1} r_{1}^{2}+-4 r_{1}^{3}+15 p_{1}^{2}+30 p_{1} r_{1}+15 r_{1}^{2}-10 p_{1}-10 r_{1}}{\left(r_{1}+1\right)^{4}},
\end{aligned}
$$

$$
\begin{aligned}
F_{r_{1}, r_{2}}\left(e_{0,4} ; x, y\right) & =y^{4}+\left(\frac{p_{2}^{4}+4 p_{2}^{3} r_{2}+6 p_{2}^{2} r_{2}^{2}+4 p_{2} r_{2}^{3}-6 p_{2}^{3}-6 r_{2}^{3}-18 p_{2} r_{2}^{2}-18 p_{2}^{2} r_{2}}{r_{2}^{4}}\right. \\
& \left.+\frac{11 p_{2}^{2}+22 p_{2} r_{2}+11 r_{2}^{2}-6 p_{2}-6 r_{2}}{r_{2}^{4}}\right) y^{4} \\
& +\frac{4\left(p_{2}-1\right)\left(r_{2}+p_{2}\right)\left(r_{2}+p_{2}-1\right)\left(r_{2}+p_{2}-2\right)}{r_{2}^{3}\left(r_{2}+1\right)} y^{3} \\
& +\frac{2\left(r_{2}+p_{2}\right)\left(r_{2}+p_{2}-1\right)\left(3 p_{2}^{2}-3 p_{2}-3 r_{2}+8\right)}{r_{2}^{2}\left(r_{2}+1\right)^{2}} y^{2} \\
& +\frac{4\left(r_{2}+p_{2}\right)\left(p_{2}^{3}-3 p_{2} r_{2}-3 r_{2}^{2}+7 p_{2}+9 r_{2}-6\right)}{r_{2}\left(r_{2}+1\right)^{3}} y \\
& +\frac{p_{2}^{4}+2 p_{2}^{3}-6 p_{2}^{2} r_{2}-12 p_{2} r_{2}^{2}+-4 r_{2}^{3}+15 p_{2}^{2}+30 p_{2} r_{2}+15 r_{2}^{2}-10 p_{2}-10 r_{2}}{\left(r_{2}+1\right)^{4}} .
\end{aligned}
$$

Proof. The proof of the above equalities can be reached easily as a consequence of Lemma 1 and by (29); hence, we have omitted the details.

Corollary 8. In view of Lemma 7, the following relations hold true:

$$
\begin{aligned}
& F_{r_{1}, r_{2}}\left(t_{0}-x ; x, y\right)=\frac{p_{1}\left(\left(r_{1}+1\right) x+r_{1}\right)}{r_{1}\left(r_{1}+1\right)}, \\
& F_{r_{1}, r_{2}}\left(s_{0}-y ; x, y\right)=\frac{p_{2}\left(\left(r_{2}+1\right) y+r_{2}\right)}{r_{2}\left(r_{2}+1\right)}, \\
& F_{r_{1}, r_{2}}\left(\left(t_{0}-x\right)^{2} ; x, y\right)=\frac{\left(p_{1}^{2}-p_{1}-r_{1}\right)}{r_{1}^{2}} x^{2}+\frac{2 p_{1}^{2}}{r_{1}\left(r_{1}+1\right)} x+\frac{p_{1}^{2}+p_{1}+r_{1}}{\left(r_{1}+1\right)^{2}}, \\
& F_{r_{1}, r_{2}}\left(\left(s_{0}-y\right)^{2} ; x, y\right)=\frac{\left(p_{2}^{2}-p_{2}-r_{2}\right)}{r_{2}^{2}} y^{2}+\frac{2 p_{2}^{2}}{r_{2}\left(r_{2}+1\right)} y+\frac{p_{2}^{2}+p_{2}+r_{2}}{\left(r_{2}+1\right)^{2}} \text {, } \\
& F_{r_{1}, r_{2}}\left(\left(t_{0}-x\right)^{4} ; x, y\right) \\
& =\frac{\left(p_{1}^{4}-6 p_{1}^{3}-6 p_{1}^{2} r_{1}-12 p_{1} r_{1}^{2}+11 p_{1}^{2}+14 p_{1} r_{1}+3 r_{1}^{2}-6 p_{1}-6 r_{1}\right)}{r_{1}^{4}} x^{4} \\
& +\frac{4\left(p_{1}^{4}-4 p_{1}^{3}-6 p_{1}^{2} r_{1}-3 p_{1} r_{1}^{2}+5 p_{1}^{2}+8 p_{1} r_{1}+3 r_{1}^{2}-2 p_{1}-2 r_{1}\right)}{r_{1}^{3}\left(r_{1}+1\right)} x^{3} \\
& +\frac{2\left(3 p_{1}^{4}-6 p_{1}^{3}-18 p_{1}^{2}-18 p_{1} r_{1}^{2}+11 p_{1}^{2}+26 p_{1} r_{1}+15 r_{1}^{2}-8 p_{1}-8 r_{1}\right)}{r_{1}^{2}\left(r_{1}+1\right)^{2}} x^{2} \\
& +\frac{4\left(p_{1}^{4}-6 p_{1}^{2} r_{1}-9 p_{1} r_{1}^{2}+7 p_{1}^{2}+16 p_{1} r_{1}+9 r_{1}^{2}-6 p_{1}-6 r_{1}\right)}{r_{1}\left(r_{1}+1\right)^{3}} x \\
& +\frac{p_{1}^{4}+2 p_{1}^{3}-6 p_{1}^{2} r_{1}-12 p_{1} r_{1}^{2}+15 p_{1}^{2}+30 p_{1} r_{1}+15 r_{1}^{2}-10 p_{1}-10 r_{1}}{\left(r_{1}+1\right)^{4}}, \\
& F_{r_{1}, r_{2}}\left(\left(s_{0}-y\right)^{4} ; x, y\right) \\
& =\frac{\left(p_{2}^{4}-6 p_{2}^{3}-6 p_{2}^{2} r_{2}-12 p_{2} r_{2}^{2}+11 p_{2}^{2}+14 p_{2} r_{2}+3 r_{2}^{2}-6 p_{2}-6 r_{2}\right)}{r_{2}^{4}} y^{4} \\
& +\frac{4\left(p_{2}^{4}-4 p_{2}^{3}-6 p_{2}^{2} r_{2}-3 p_{2} r_{2}^{2}+5 p_{2}^{2}+8 p_{2} r_{2}+3 r_{2}^{2}-2 p_{2}-2 r_{2}\right)}{r_{2}^{3}\left(r_{2}+1\right)} y^{3} \\
& +\frac{2\left(3 p_{2}^{4}-6 p_{2}^{3}-18 p_{2}^{2}-18 p_{2} r_{2}^{2}+11 p_{2}^{2}+26 p_{2} r_{2}+15 r_{2}^{2}-8 p_{2}-8 r_{2}\right)}{r_{2}^{2}\left(r_{2}+1\right)^{2}} y^{2} \\
& +\frac{4\left(p_{2}^{4}-6 p_{2}^{2} r_{2}-9 p_{2} r_{2}^{2}+7 p_{2}^{2}+16 p_{2} r_{2}+9 r_{2}^{2}-6 p_{2}-6 r_{2}\right)}{r_{2}\left(r_{2}+1\right)^{3}} y \\
& +\frac{p_{2}^{4}+2 p_{2}^{3}-6 p_{2}^{2} r_{2}-12 p_{2} r_{2}^{2}+15 p_{2}^{2}+30 p_{2} r_{2}+15 r_{2}^{2}-10 p_{2}-10 r_{2}}{\left(r_{2}+1\right)^{4}} .
\end{aligned}
$$


Theorem 9. Let the operators $F_{r_{1}, r_{2}}(\mu ; x, y)$ be given by (29). Then for any $\mu \in C\left(D_{r_{1}, r_{2}}\right)$, we arrive at

$$
\lim _{r_{1}, r_{2} \longrightarrow \infty}\left\|F_{r_{1}, r_{2}}(\mu)-\mu\right\|=0
$$

Proof. It is seen from the following that

$$
\begin{aligned}
& \left\|F_{r_{1}, r_{2}}\left(e_{0,0}\right)-e_{0,0}\right\| \longrightarrow 0,\left\|F_{r_{1}, r_{2}}\left(e_{1,0}\right)-e_{1,0}\right\| \longrightarrow 0, \\
& \left\|F_{r_{1}, r_{2}}\left(e_{0,1}\right)-e_{0,1}\right\| \longrightarrow 0,\left\|F_{r_{1}, r_{2}}\left(e_{2,0}+e_{0,2}\right)-\left(e_{2,0}+e_{0,2}\right)\right\| \longrightarrow 0,
\end{aligned}
$$

as $r_{1}, r_{2} \longrightarrow \infty$. Thus, these results complete the proof, as required by the Volkov theorem [26].

Moreover, for the operators given by (29), we want to derive the Voronovskaya-type asymptotic theorem and estimate the degree of convergence with the help of Peetre's $K$-functional.

Suppose that $C^{2}\left(D_{r_{1}, r_{2}}\right)$ represents the space of all functions of $\mu \in C\left(D_{r_{1}, r_{2}}\right)$ such that $\partial_{i} \mu / \partial x_{i}, \partial_{i} \mu / \partial y_{i} \in C\left(D_{r_{1}, r_{2}}\right)$ for $i=1,2)$. The norm on $C^{2}\left(D_{r_{1}, r_{2}}\right)$ and Peetre's $K$-functional of $\mu \in C\left(D_{r_{1}, r_{2}}\right)$ are given as follows, respectively.

$\|\mu\|_{C^{2}\left(D_{r_{1}, r_{2}}\right)}=\|\mu\|_{C\left(D_{r_{1}, r_{2}}\right)}+\sum_{i=1}^{2}\left(\left\|\frac{\partial_{i} \mu}{\partial x_{i}}\right\|_{C\left(D_{r_{1}, r_{2}}\right)}+\left\|\frac{\partial_{i} \mu}{\partial y_{i}}\right\|_{C\left(D_{r_{1}, r_{2}}\right)}\right)$,

$K(\mu, \zeta)=\inf \left\{\|\mu-\mathrm{Q}\|_{C\left(D_{r_{1}, r_{2}}\right)}+\zeta\|\mathrm{\varrho}\|_{C^{2}\left(D_{r_{1}, r_{2}}\right)}: \mathrm{\varrho} \in C^{2}\left(D_{r_{1}, r_{2}}\right)\right\}$,

where $\zeta>0$.

For a constant $C>0$, the following inequality

$$
K(\mu, \zeta) \leq C \omega_{2}^{*}(\mu, \sqrt{\zeta})
$$

holds, where $\omega_{2}{ }^{*}(\mu, \sqrt{\zeta})$ denotes the second order of the modulus of continuity of $\mu \in C\left(D_{r_{1}, r_{2}}\right)$. Also, for $\mu \in C\left(D_{r_{1}, r_{2}}\right)$, the ordinary modulus of continuity is defined as

$$
\begin{aligned}
\omega(\mu, \delta) & =\sup \left\{\left|\mu\left(u_{1}, u_{2}\right)-\mu\left(v_{1}, v_{2}\right)\right|:\left(u_{1}, u_{2}\right),\left(v_{1}, v_{2}\right)\right. \\
& \left.\in C\left(D_{r_{1}, r_{2}}\right), \sqrt{\left(u_{1}-v_{1}\right)^{2}+\left(u_{2}-v_{2}\right)^{2}} \leq \delta\right\} .
\end{aligned}
$$

Theorem 10. Suppose that $\mu \in C^{2}\left(D_{r_{1}, r_{2}}\right)$. Then, the following relation holds

$$
\begin{aligned}
\lim _{r_{1} \longrightarrow \infty} & r_{1}\left[F_{r_{1}, r_{1}}(\mu ; x, y)-\mu ; x, y\right] \\
= & p_{1}(x+1) \mu_{x}^{\prime}(x, y)+p_{2}(y+1) \mu_{y}^{\prime}(x, y)+\frac{1}{2} \\
& \times\left\{\left(1-x^{2}\right) \mu_{x x}^{\prime \prime}(x, y)+\left(1-y^{2}\right) \mu_{y y}^{\prime \prime}(x, y)\right\},
\end{aligned}
$$

uniformly on $D_{r_{1}, r_{2}}$.
Proof. For the arbitrary $(x, y) \in D_{r_{1}, r_{2}}$, using the Taylor formula, it becomes

$$
\begin{aligned}
\mu\left(t_{0}, s_{0}\right)= & \mu(x, y)+\mu_{x}^{\prime}(x, y)\left(t_{0}-x\right)+\mu_{y}^{\prime}(x, y)\left(s_{0}-y\right)+\frac{1}{2} \\
& \cdot\left\{\mu_{x x}^{\prime \prime}(x, y)\left(t_{0}-x\right)^{2}+\mu_{y y}^{\prime \prime}(x, y)\left(s_{0}-y\right)^{2}+2 \mu_{x y}^{\prime \prime}\right. \\
& \left.\cdot(x, y)\left(t_{0}-x\right)\left(s_{0}-y\right)\right\}+\chi\left(t_{0}, s_{0} ; x, y\right) \\
& \cdot \sqrt{\left(t_{0}-x\right)^{4}+\left(s_{0}-y\right)^{4}}
\end{aligned}
$$

for $\left(t_{0}, s_{0}\right) \in D_{r_{1}, r_{2}}$, where $\chi(., . ; x, y) \in C\left(D_{r_{1}, r_{2}}\right)$ and $\chi\left(t_{0}, s_{0}\right.$; $x, y) \longrightarrow 0$, as $\left(t_{0}, s_{0}\right) \longrightarrow(x, y)$.

Operating $F_{r_{1}, r_{1}}(. ; x, y)$ on (38) yields

$$
\begin{aligned}
F_{r_{1}, r_{1}}( & \left.\mu\left(t_{0}, s_{0}\right) ; x, y\right) \\
= & \mu(x, y)+\mu_{x}^{\prime}(x, y) F_{r_{1}, r_{1}}\left(t_{0}-x ; x, y\right)+\mu_{y}^{\prime}(x, y) F_{r_{1}, r_{1}} \\
& \times\left(s_{0}-y ; x, y\right)+\frac{1}{2}\left\{\mu_{x x}^{\prime \prime}(x, y) F_{r_{1}, r_{1}}\left(\left(t_{0}-x\right)^{2} ; x, y\right)\right. \\
& +\mu_{y y}^{\prime \prime}(x, y) F_{r_{1}, r_{1}}\left(\left(s_{0}-y\right)^{2} ; x, y\right)+2 \mu_{x y}^{\prime \prime}(x, y) F_{r_{1}, r_{1}} \\
& \left.\times\left(\left(t_{0}-x\right)\left(s_{0}-y\right) ; x, y\right)\right\}+F_{r_{1}, r_{1}} \\
& \times\left(\chi\left(t_{0}, s_{0} ; x, y\right) \sqrt{\left(t_{0}-x\right)^{4}+\left(s_{0}-y\right)^{4}} ; x, y\right) .
\end{aligned}
$$

If we use the Cauchy-Schwarz inequality to the last part of (39), one has

$$
\begin{aligned}
\mid F_{r_{1}, r_{1}} & \left(\chi\left(t_{0}, s_{0} ; x, y\right) \sqrt{\left(t_{0}-x\right)^{4}+\left(s_{0}-y\right)^{4}} ; x, y\right) \mid \\
\leq & \sqrt{F_{r_{1}, r_{1}}\left(\chi^{2}\left(t_{0}, s_{0} ; x, y\right) ; x, y\right)} \\
& \times \sqrt{F_{r_{1}, r_{1}}\left(\left(t_{0}-x\right)^{4} ; x, y\right)+F_{r_{1}, r_{1}}\left(\left(s_{0}-y\right)^{4} ; x, y\right)} .
\end{aligned}
$$

Considering Theorem 9 and because of $\chi(., . ; x, y) \in C$ $\left(D_{r_{1}, r_{2}}\right), \chi\left(t_{0}, s_{0} ; x, y\right) \longrightarrow 0$, as $\left(t_{0}, s_{0}\right) \longrightarrow(x, y)$, then

$$
\lim _{r_{1} \longrightarrow \infty} F_{r_{1}, r_{1}}\left(\chi^{2}\left(t_{0}, s_{0} ; x, y\right) ; x, y\right)=0,
$$

uniformly on $D_{r_{1}, r_{2}}$. Also, from Corollary 8, it is clear to see

$$
\lim _{r_{1} \longrightarrow \infty} r_{1}\left[F_{r_{1}, r_{1}}\left(\left(t_{0}-x\right)\left(s_{0}-y\right) ; x, y\right)\right]=0 .
$$

Further, by Corollary 3, it follows that

$$
\begin{aligned}
& \lim _{r_{1} \longrightarrow \infty} r_{1}^{2}\left[F_{r_{1}, r_{1}}\left(\left(t_{0}-x\right)^{4} ; x, y\right)\right]=3\left(1-3 x^{2}\right)\left(x^{2}+1\right), \\
& \lim _{1} r_{1}^{2}\left[F_{r_{1}, r_{1}}\left(\left(s_{0}-y\right)^{4} ; x, y\right)\right]=3\left(1-3 y^{2}\right)\left(y^{2}+1\right) .
\end{aligned}
$$


Thus,

$\lim _{r_{1} \longrightarrow \infty} r_{1}\left[F_{r_{1}, r_{1}}\left(\chi\left(t_{0}, s_{0} ; x, y\right) \sqrt{\left(t_{0}-x\right)^{4}+\left(s_{0}-y\right)^{4}} ; x, y\right)\right]=0$.

Hence, the desired sequel is arrived as follows:

$$
\begin{aligned}
\lim _{r_{1} \rightarrow \infty} & r_{1}\left[F_{r_{1}, r_{1}}(\mu ; x, y)-\mu ; x, y\right] \\
= & p_{1}(x+1) \mu_{x}^{\prime}(x, y)+p_{2}(y+1) \mu_{y}^{\prime}(x, y)+\frac{1}{2}\left\{\left(1-x^{2}\right) \mu_{x x}^{\prime \prime}\right. \\
& \left.\times(x, y)+\left(1-y^{2}\right) \mu_{y y}^{\prime \prime}(x, y)\right\},
\end{aligned}
$$

uniformly on $D_{r_{1}, r_{2}}$.

Theorem 11. Suppose that $\mu \in C\left(D_{r_{1}, r_{2}}\right)$. Then, the following inequality is verified:

$$
\begin{aligned}
& \left|F_{r_{1}, r_{2}}(\mu ; x, y)-\mu(x, y)\right| \\
& \leq C_{1}\left\{\omega_{2}^{*}\left(\mu ; \sqrt{S_{r_{1}, r_{2}}(x, y)}\right)+\min \left\{1, S_{r_{1}, r_{2}}(x, y)\|\mu\|_{C^{2}\left(D_{r_{1}, r_{2}}\right)}\right\}\right. \\
& +\omega\left(\mu ; \xi_{r_{1}, r_{2}}(x, y)\right),
\end{aligned}
$$

where $C_{1}>0$ is a constant and independent of $\mu$ and $S_{r_{1}, r_{2}}(x, y)$ and $\gamma_{r_{1}}^{2}(x)=F_{r_{1}, r_{2}}\left(\left(t_{0}-x\right)^{2} ; x, y\right), \gamma_{r_{2}}^{2}(y)=F_{r_{1}, r_{2}}\left(\left(s_{0}-y\right)^{2} ; x\right.$, $\left.\left.\left.y), \xi_{r_{1}, r_{2}}(x, y)=\sqrt{\left(p_{1}((\right.} r_{1}+1\right) x+r_{1}\right) / r_{1}\left(r_{1}+1\right)\right)^{2}+\left(p_{2}\left(\left(r_{2}\right.\right.\right.$ $\left.\left.+1) y+r_{2}\right) / r_{2}\left(r_{2}+1\right)\right)^{2}, S_{r_{1}, r_{2}}(x, y)=\gamma_{r_{1}}^{2}(x)+\gamma_{r_{2}}^{2}(y)+\xi_{r_{1}, r_{2}}^{2}$.

Proof. Let us define the following auxiliary operators:

$$
\begin{aligned}
F_{r_{1}, r_{2}}^{-}(\mu ; x, y)= & F_{r_{1}, r_{2}}(\mu ; x, y)+\mu(x, y)-\mu \\
& \cdot\left(x+\frac{p_{1}\left(\left(r_{1}+1\right) x+r_{1}\right)}{r_{1}\left(r_{1}+1\right)}, y+\frac{p_{2}\left(\left(r_{2}+1\right) y+r_{2}\right)}{r_{2}\left(r_{2}+1\right)}\right) .
\end{aligned}
$$

By Lemma 7, we obtain

$$
\begin{aligned}
& F_{r_{1}, r_{2}}^{-}\left(t_{0}-x ; x, y\right)=0, \\
& F_{r_{1}, r_{2}}^{-}\left(s_{0}-y ; x, y\right)=0 .
\end{aligned}
$$

Suppose that $\mu \in C^{2}\left(D_{r_{1}, r_{2}}\right)$ and $\left(t_{0}, s_{0}\right) \in D_{r_{1}, r_{2}}$; by the Taylor formula, we may write

$$
\begin{aligned}
\mu\left(t_{0}, s_{0}\right)-\mu(x, y)= & \frac{\partial \mu(x, y)}{\partial x}\left(t_{0}-x\right)+\int_{x}^{t_{0}}\left(t_{0}-u\right) \frac{\partial^{2} \mu(u, y)}{\partial^{2} u} d u \\
& +\frac{\partial \mu(x, y)}{\partial y}\left(s_{0}-y\right)+\int_{y}^{s_{0}}\left(s_{0}-v\right) \frac{\partial^{2} \mu(x, v)}{\partial^{2} v} d v .
\end{aligned}
$$

Now, operating $F_{r_{1}, r_{2}}^{-}$on (49), we get

$$
\begin{aligned}
& F_{r_{1}, r_{2}}^{-}(\mu ; x, y)-\mu(x, y) \\
&=F_{r_{1}, r_{2}}^{-}\left(\int_{x}^{t_{0}}\left(t_{0}-u\right) \frac{\partial^{2} \mu(u, y)}{\partial^{2} u} d u ; x, y\right) \\
& \quad+F_{r_{1}, r_{2}}^{-}\left(\int_{y}^{s_{0}}\left(s_{0}-v\right) \frac{\partial^{2} \mu(x, v)}{\partial^{2} v} d v ; x, y\right) \\
&=F_{r_{1}, r_{2}}\left(\int_{x}^{t_{0}}\left(t_{0}-u\right) \frac{\partial^{2} \mu(u, y)}{\partial^{2} u} d u ; x, y\right) \\
&-\int_{x}^{\left.x+\left(p_{1}\left(\left(r_{1}+1\right) x+r_{1}\right)\right) r_{1}\left(r_{1}+1\right)\right)}\left(x+\frac{p_{1}\left(\left(r_{1}+1\right) x+r_{1}\right)}{r_{1}\left(r_{1}+1\right)}-u\right) \\
& \quad \times \frac{\partial^{2} \mu(u, y)}{\partial^{2} u} d u+F_{r_{1}, r_{2}}\left(\int_{y}^{s_{0}}\left(s_{0}-v\right) \frac{\partial^{2} \mu(x, v)}{\partial^{2} v} d v ; x, y\right) \\
&- \int_{y}^{\left.y+\left(p_{2}\left(\left(r_{2}+1\right) y+r_{2}\right)\right) r_{2}\left(r_{2}+1\right)\right)}\left(y+\frac{p_{2}\left(\left(r_{2}+1\right) y+r_{2}\right)}{r_{2}\left(r_{2}+1\right)}-v\right) \\
& \times \frac{\partial^{2} \mu(x, v)}{\partial^{2} v} d v .
\end{aligned}
$$

\section{Hence,}

$$
\begin{aligned}
\left|F_{r_{1}, r_{2}}(\mu ; x, y)-\mu(x, y)\right| & \leq\left(\left|\int_{x}^{t_{0}}\right| t_{0}-u|| \frac{\partial^{2} \mu(u, y)}{\partial^{2} u}|d u| ; x, y\right) \\
\leq & F_{r_{1}, r_{2}}\left(\int_{x}^{x+\left(p_{1}\left(\left(r_{1}+1\right) x+r_{1}\right) / r_{1}\left(r_{1}+1\right)\right)}\left|x+\frac{p_{1}\left(\left(r_{1}+1\right) x+r_{1}\right)}{r_{1}\left(r_{1}+1\right)}-u\right|\right. \\
& \times\left|\frac{\partial^{2} \mu(u, y)}{\partial^{2} u}\right| d u \mid+F_{r_{1}, r_{2}}\left(\left|\int_{y}^{s_{0}}\right| s_{0}-v|| \frac{\partial^{2} \mu(x, v)}{\partial^{2} v}|d v| ; x, y\right) \\
& +\left|\int_{y}^{\left.y+\left(p_{2}\left(\left(r_{2}+1\right) y+r_{2}\right)\right) r_{2}\left(r_{2}+1\right)\right)}\right| y+\frac{p_{2}\left(\left(r_{2}+1\right) y+r_{2}\right)}{r_{2}\left(r_{2}+1\right)}-v \mid \\
& \times\left|\frac{\partial^{2} \mu(x, v)}{\partial^{2} v}\right| d v \mid \\
\leq & \left\{F_{r_{1}, r_{2}}\left(\left(t_{0}-x\right)^{2} ; x, y\right)+\left(x+\frac{p_{1}\left(\left(r_{1}+1\right) x+r_{1}\right)}{r_{1}\left(r_{1}+1\right)}-x\right)^{2}\right. \\
& \left.+F_{r_{1}, r_{2}}\left(\left(s_{0}-y\right)^{2} ; x, y\right)+\left(y+\frac{p_{2}\left(\left(r_{2}+1\right) y+r_{2}\right)}{r_{2}\left(r_{2}+1\right)}-y\right)^{2}\right\} \\
& \times\|\mu\|_{C^{2}\left(D_{r_{1}, r_{2}}\right)} .
\end{aligned}
$$


$S_{r_{1}, r_{2}}(x, y)=\gamma_{r_{1}}^{2}(x)+\gamma_{r_{2}}^{2}(y)+\xi_{r_{1}, r_{2}}^{2}$, then

$$
\left|F_{r_{1}, r_{2}}(\mu ; x, y)-\mu(x, y)\right| \leq S_{r_{1}, r_{2}}(x, y)\|\mu\|_{C^{2}\left(D_{r_{1}, r_{2}}\right)} .
$$

Also, by Lemma 7 and (52), we arrive at

$$
\begin{aligned}
\left|F_{r_{1}, r_{2}}^{-}(\mu ; x, y)\right| \leq & \left|F_{r_{1}, r_{2}}(\mu ; x, y)\right|+|\mu(x, y)| \\
& +\mid \mu\left(x+\frac{p_{1}\left(\left(r_{1}+1\right) x+r_{1}\right)}{r_{1}\left(r_{1}+1\right)}, y\right. \\
& \left.+\frac{p_{2}\left(\left(r_{2}+1\right) y+r_{2}\right)}{r_{2}\left(r_{2}+1\right)}\right) \mid \\
\leq & 3\|\mu\|_{C\left(D_{r_{1}, r_{2}}\right)} .
\end{aligned}
$$

Next, from (53)

$$
\begin{aligned}
& \left|F_{r_{1}, r_{2}}(\mu ; x, y)-\mu(x, y)\right| \\
& \leq \mid \\
& \quad+\left|F_{r_{1}, r_{2}}^{-}(\mu-\kappa ; x, y)\right|+\left|F_{r_{1}, r_{2}}^{-}(\kappa ; x, y)-\kappa(x, y)\right| \\
& \left.\quad+\mid x+\frac{p_{1}\left(\left(r_{1}+1\right) x+r_{1}\right)}{r_{1}\left(r_{1}+1\right)}, y+\frac{p_{2}\left(\left(r_{2}+1\right) y+r_{2}\right)}{r_{2}\left(r_{2}+1\right)}\right) \\
& \quad-\mu(x, y)|+| \kappa(x, y)-\mu(x, y \mid \\
& \leq \quad\left(4\|\mu-\kappa\|_{C\left(D_{r_{1}, r_{2}}\right)}+S_{r_{1}, r_{2}}(x, y)\|\kappa\|_{C^{2}\left(D_{r_{1}, r_{2}}\right)}\right) \\
& \quad+\omega\left(\mu ; \xi_{r_{1}, r_{2}}(x, y)\right) .
\end{aligned}
$$

Consequently, on (54), utilizing the infimum on the righthand side over all $\mu \in C^{2}\left(D_{r_{1}, r_{2}}\right)$ and by (35), it becomes

$$
\begin{aligned}
& \left|F_{r_{1}, r_{2}}(\mu ; x, y)-\mu(x, y)\right| \\
& \leq C_{1}\left\{\omega_{2}^{*}\left(\mu ; \sqrt{S_{r_{1}, r_{2}}(x, y)}\right)+\min \left\{1, S_{r_{1}, r_{2}}(x, y)\|\mu\|_{C^{2}\left(D_{r_{1}, r_{2}}\right)}\right\}\right. \\
& \quad+\omega\left(\mu ; \xi_{r_{1}, r_{2}}(x, y)\right),
\end{aligned}
$$

which ends the proof.

\section{Construction of the GBS Type of $F_{r_{1}, r_{2}}(\mu ; x, y)$}

The notion of the $B$-continuous and $B$-differentiable functions was firstly used by Bögel $[27,28]$. Dobrescu and Matei [29] considered the GBS (Generalized Boolean Sum) kind of the bivariate of the Bernstein polynomials. Next, using the $B$-continuous functions by the GBS operators, which is related to quantitative version of the Korovkin-type convergence theorem, firstly has been improved by Badea et al. [30, 31]. Pop and Fărcas [32] obtained some approximation of the $B$-continuous and $B$-differentiable functions by GBS type of the Bernstein bivariate operators. İspir [33] established quantitative estimates for the GBS of the Chlodowsky-Szász-kind operators. Recently, some authors introduced the GBS operators of various operators (we refer the readers to [34-42]).
Let a function $\mu: U \times V \longrightarrow \mathbb{R}$, where $U, V$ are compact real intervals of $\mathbb{R}$. With $(x, y),\left(t_{0}, s_{0}\right) \in U \times V$, the mixed difference of the function $\mu$ is given as

$$
\phi_{(x, y)} \mu\left[t_{0}, s_{0} ; x, y\right]=\mu(x, y)-\mu\left(x, s_{0}\right)-\mu\left(t_{0}, y\right)+\mu\left(t_{0}, s_{0}\right) .
$$

A function $\mu: U \times V \longrightarrow \mathbb{R}$ is named Bögel-continuous (B-continuous) at $\left(t_{0}, s_{0}\right) \in U \times V$, if

$$
\lim _{(x, y) \longrightarrow\left(t_{0}, s_{0}\right)} \phi_{(x, y)} \mu\left[t_{0}, s_{0} ; x, y\right]=0 .
$$

A function $\mu: U \times V \longrightarrow \mathbb{R}$ is named Bögeldifferentiable (B-differentiable) at $\left(t_{0}, s_{0}\right) \in U \times V$, if the below result which denoted by $D_{B} f(x, y)$ exists and finite

$$
\lim _{(x, y) \longrightarrow\left(t_{0}, s_{0}\right)} \frac{\phi_{(x, y)} \mu\left[t_{0}, s_{0} ; x, y\right]}{\left(x-t_{0}\right)\left(y-s_{0}\right)}=D_{B} f(x, y) .
$$

Note that, by $C_{b}(U \times V)$ and $D_{b}(U \times V)$, we represent the sets of all $B$-differentiable and $B$-continuous functions on $U \times V$, respectively.

A function $\mu: Y \subset U \times V \longrightarrow \mathbb{R}$ is named Bögel-bounded (B-bounded) on $Y$, if there consists $W>0$ such that $\mid \phi_{(x, y)}$ $\mu\left[t_{0}, s_{0} ; x, y\right] \mid \leq W$ for every $\left(t_{0}, s_{0}\right),(x, y) \in Y$.

Also, if $Y$ is a compact subset of $\mathbb{R}^{2}$, hence all $B$-continuous functions are $B-$ bounded on $Y \longrightarrow \mathbb{R}$.

Further, by $B_{b}(Y)$, we represent the set of all $B$-bounded functions on $Y$ and equipped with the norm $\|\mu\|_{B}=$

$\left.\sup _{\sup } \mid \phi_{(x, y)} \mu\left[t_{0}, s_{0} ; x, y\right]\right) \mid$. Considering the definition of $B$-continuous, then $C(Y) \subset C_{b}(Y)$ (see details by [43]).

The mixed modulus of smoothness for $\mu \in C_{b}\left(D_{r_{1}, r_{2}}\right)$ is given by

$$
\begin{aligned}
& \omega_{\text {mixed }}\left(\mu ; \delta_{1}, \delta_{2}\right) \\
& \quad:=\sup \left\{\left|\phi_{(x, y)} \mu\left[t_{0}, s_{0} ; x, y\right]\right|:\left|t_{0}-x\right|<\delta_{1},\left|s_{0}-y\right|<\delta_{2}\right\},
\end{aligned}
$$

where $(x, y),\left(t_{0}, s_{0}\right) \in D_{r_{1}, r_{2}}$, and $\delta_{1}, \delta_{2} \in \mathbb{R}^{+}$. Also, for all $\kappa_{1}$, $\kappa_{2} \geq 0$, the following inequality satisfy

$$
\omega_{\text {mixed }}\left(\mu ; \kappa_{1} \delta_{1}, \kappa_{2} \delta_{2}\right) \leq\left(1+\kappa_{1}\right)\left(1+\kappa_{2}\right) \omega_{\text {mixed }}\left(\mu ; \delta_{1}, \delta_{2}\right) .
$$

More details about the mixed modulus of smoothness can be found in $[30,31]$.

Now, for all $(x, y) \in D_{r_{1}, r_{2}}$, for any $\mu \in C_{b}\left(D_{r_{1}, r_{2}}\right)$ and $\left(r_{1}\right.$, $\left.r_{2}\right) \in \mathbb{N} \times \mathbb{N},\left(p_{1}, p_{2}\right) \in \mathbb{N}_{0} \times \mathbb{N}_{0}$, we construct the GBS-type operators given by (29) operators as follows:

$$
G_{r_{1}, r_{2}}(\mu ; x, y)=F_{r_{1}, r_{2}}\left(\mu\left(x, s_{0}\right)+\mu\left(t_{0}, y\right)-\mu\left(t_{0}, s_{0}\right) ; x, y\right) .
$$


Exactly, for any $\mu \in C_{b}\left(D_{r_{1}, r_{2}}\right)$ and for all $(x, y) \in D_{r_{1}, r_{2}}$, the GBS type of $F_{r_{1}, r_{2}}$ operators is given as

$$
\begin{aligned}
G_{r_{1}, r_{2}}(\mu ; x, y)= & \left(\frac{r_{1}+1}{2 r_{1}}\right)^{r_{1}+p_{1}}\left(\frac{r_{2}+1}{2 r_{2}}\right)^{r_{2}+p_{2}} \sum_{j_{1}=0}^{r_{1}+p_{1}} \sum_{j_{2}=0}^{r_{2}+p_{2}} \varphi_{r_{1}, r_{2}, j_{1}, j_{2}}^{p_{1}, p_{2}} \\
& \cdot(x, y) \times\left(\mu\left(x, \frac{2 j_{2}-r_{2}}{r_{2}+1}\right)+\mu\left(\frac{2 j_{1}-r_{1}}{r_{1}+1}, y\right)\right. \\
& \left.-\mu\left(\frac{2 j_{1}-r_{1}}{r_{1}+1}, \frac{2 j_{2}-r_{2}}{r_{2}+1}\right)\right),
\end{aligned}
$$

where $\varphi_{r_{1}, r_{2}, j_{1}, j_{2}}^{p_{1}, p_{2}}(x, y)$ is defined as in (29).

It is clear that the operators given by (62) are linear and positive. In the following theorem, with regard to the mixed modulus of smoothness, we estimate the order of approximation of the (62) operators.

Theorem 12. For all $\mu \in C_{b}\left(D_{r_{1}, r_{2}}\right)$ and for each $(x, y) \in D_{r_{1}, r_{2}}$, the operators given by (62) satisfy the following inequality:

$$
\left|G_{r_{1}, r_{2}}(\mu ; x, y)-\mu(x, y)\right| \leq 4 \omega_{\text {mixed }}\left(\mu ; \sqrt{\left(\alpha_{r_{1}, p_{1}}\right)}, \sqrt{\left(\alpha_{r_{2}, p_{2}}\right)}\right),
$$

where $_{r_{1}, p_{1}}=\left(p_{1}^{2}-3 p_{1}+r_{1}+4\right) /\left(r_{1}+1\right)^{2}$ and $\alpha_{r_{2}, p_{2}}=\left(p_{2}^{2}-3\right.$ $\left.p_{2}+r_{2}+4\right) /\left(r_{2}+1\right)^{2}$.

Proof. In view of (60), it gives

$$
\begin{aligned}
\left|\phi_{(x, y)} \mu\left[t_{0}, s_{0} ; x, y\right]\right| \leq & \omega_{\text {mixed }}\left(\mu ;\left|t_{0}-x\right|,\left|s_{0}-y\right|\right) \\
\leq & \left(1+\frac{\left|t_{0}-x\right|}{\delta_{1}}\right)\left(1+\frac{\left|s_{0}-y\right|}{\delta_{2}}\right) \omega_{\text {mixed }} \\
& \cdot\left(\mu ; \delta_{1}, \delta_{2}\right),
\end{aligned}
$$

for all $(x, y),\left(t_{0}, s_{0}\right) \in D_{r_{1}, r_{2}}$, and for any $\delta_{1}, \delta_{2} \in \mathbb{R}^{+}$. By (56), we have

$$
\mu\left(x, s_{0}\right)+\mu\left(t_{0}, y\right)-\mu\left(t_{0}, s_{0}\right)=\mu(x, y)-\phi_{(x, y)} \mu\left[t_{0}, s_{0} ; x, y\right] .
$$

Operating $F_{r_{1}, r_{2}}$ and by the definition of $G_{r_{1}, r_{2}}$, then

$$
\begin{aligned}
G_{r_{1}, r_{2}}(\mu ; x, y)= & \mu(x, y) F_{r_{1}, r_{2}}\left(e_{0,0} ; x, y\right)-F_{r_{1}, r_{2}} \\
& \cdot\left(\phi_{(x, y)} \mu\left[t_{0}, s_{0} ; x, y\right] ; x, y\right) .
\end{aligned}
$$

It follows that

$$
\left|G_{r_{1}, r_{2}}(\mu ; x, y)-\mu(x, y)\right| \leq F_{r_{1}, r_{2}}\left(\left|\phi_{(x, y)} \mu\left[t_{0}, s_{0} ; x, y\right]\right| ; x, y\right) .
$$

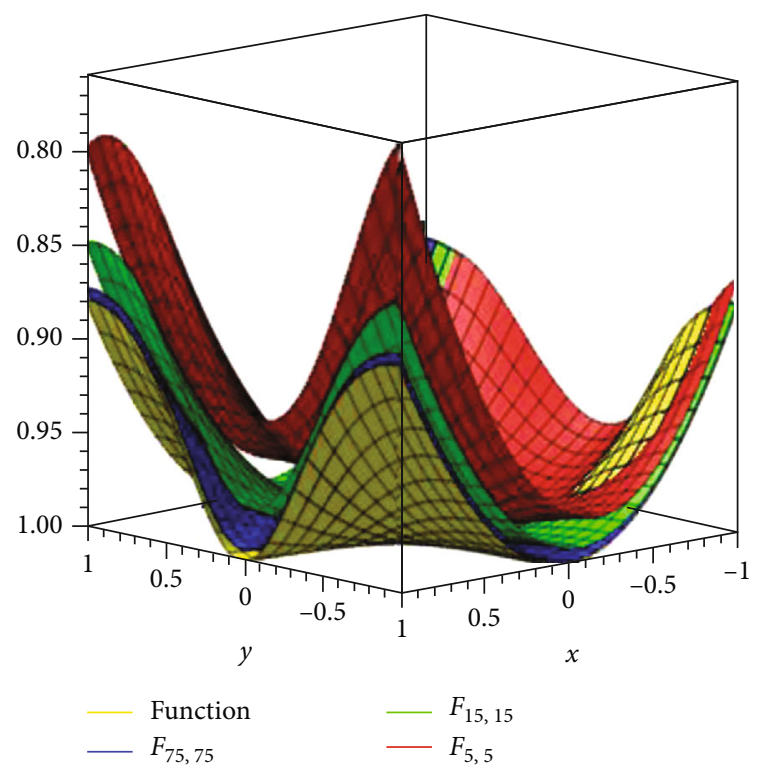

FIGURE 1: The convergence of $F_{r_{1}, r_{2}}(\mu ; x, y)$ operators to $\mu(x, y)=$ $\cos \left(x y /\left(1+y^{2}\right)\right)$ (yellow) for $r_{1}=r_{2}=5$ (red), $r_{1}=r_{2}=15$ (green), $r_{1}=r_{2}=75$ (blue), and $p_{1}=p_{2}=1$.

From (64) and by utilizing the Cauchy-Schwarz inequality, one has

$$
\begin{aligned}
& \left|G_{r_{1}, r_{2}}(\mu ; x, y)-\mu(x, y)\right| \\
& \leq\left(1+\frac{\sqrt{F_{r_{1}, r_{2}}\left(\left(t_{0}-x\right)^{2} ; x, y\right)}}{\delta_{1}}+\frac{\sqrt{F_{r_{1}, r_{2}}\left(\left(s_{0}-y\right)^{2} ; x, y\right)}}{\delta_{2}}\right. \\
& \left.\quad+\frac{\sqrt{F_{r_{1}, r_{2}}\left(\left(t_{0}-x\right)^{2} ; x, y\right) F_{r_{1}, r_{2}}\left(\left(s_{0}-y\right)^{2} ; x, y\right)}}{\delta_{1} \delta_{2}}\right) \\
& \quad \times \omega_{\text {mixed }}\left(\mu ; \delta_{1}, \delta_{2}\right) .
\end{aligned}
$$

Taking into account Corollary 8 , it becomes

$$
\begin{aligned}
F_{r_{1}, r_{2}}\left(\left(t_{0}-x\right)^{2} ; x, y\right) & \leq\left(\frac{p_{1}}{r_{1}} x\right)^{2}+\frac{2 p_{1}^{2}}{r_{1}\left(r_{1}+1\right)} x+\frac{p_{1}^{2}+p_{1}+r_{1}}{\left(r_{1}+1\right)^{2}} \\
& \leq \frac{p_{1}^{2}-3 p_{1}+r_{1}+4}{\left(r_{1}+1\right)^{2}}=\alpha_{r_{1}, p_{1}}, \\
F_{r_{1}, r_{2}}\left(\left(s_{0}-y\right)^{2} ; x, y\right) & \leq\left(\frac{p_{2}}{r_{2}} y\right)^{2}+\frac{2 p_{2}^{2}}{r_{2}\left(r_{2}+1\right)} y+\frac{p_{2}^{2}+p_{2}+r_{2}}{\left(r_{2}+1\right)^{2}} \\
& \leq \frac{p_{2}^{2}-3 p_{2}+r_{2}+4}{\left(r_{2}+1\right)^{2}}=\alpha_{r_{2}, p_{2}} .
\end{aligned}
$$


TABLE 1: Error of approximation $F_{r_{1}, r_{2}}(\mu ; x, y)$ operators to $\mu(x, y)=\cos \left(x y /\left(1+y^{2}\right)\right)$ for $r_{1}=r_{2}=5, r_{1}=r_{2}=50, r_{1}=r_{2}=500$, and $p_{1}=$ $p_{2}=1$.

\begin{tabular}{lccc}
\hline$(x, y)$ & $\left|F_{5,5}(\mu ; x, y)-\mu(x, y)\right|$ & $\left|F_{50,50}(\mu ; x, y)-\mu(x, y)\right|$ & $\left|F_{500,500}(\mu ; x, y)-\mu(x, y)\right|$ \\
\hline$(0.6,0.6)$ & 0.0589662082 & 0.0057059967 & 0.0005642954 \\
$(0.8,-0.1)$ & 0.0532558351 & 0.0049072492 & 0.0004809529 \\
$(0.5,-0.5)$ & 0.0271614376 & 0.0024426683 & 0.0002455107 \\
$(0.4,-0.3)$ & 0.0203534842 & 0.0014131511 & 0.0001327585 \\
$(0.3,-0.7)$ & 0.0170542963 & 0.0010060800 & 0.0000896256 \\
$(-0.2,0.8)$ & 0.0148469930 & 0.0015555907 & 0.0001550674 \\
$(-0.6,0.4)$ & 0.0127417986 & 0.0019020417 & 0.0001971948 \\
$(-0.1,0.1)$ & 0.0091694597 & 0.0003325468 & 0.0000204350 \\
$(-0.3,-0.7)$ & 0.0081301508 & 0.0007964789 & 0.0000801801 \\
$(-0.9,0.9)$ & 0.0020362863 & 0.0003014692 & 0.0000358039 \\
\hline
\end{tabular}

Hence,

$$
\begin{aligned}
& \left|G_{r_{1}, r_{2}}(\mu ; x, y)-\mu(x, y)\right| \\
& \leq\left(1+\frac{\sqrt{\left(p_{1}^{2}-3 p_{1}+r_{1}+4\right) /\left(r_{1}+1\right)^{2}}}{\delta_{1}}+\frac{\sqrt{\left(p_{2}^{2}-3 p_{2}+r_{2}+4\right) /\left(r_{2}+1\right)^{2}}}{\delta_{2}}\right. \\
& \left.\quad+\frac{\sqrt{\left(\left(p_{1}^{2}-3 p_{1}+r_{1}+4\right) /\left(r_{1}+1\right)^{2}\right)\left(\left(p_{2}^{2}-3 p_{2}+r_{2}+4\right) /\left(r_{2}+1\right)^{2}\right)}}{\delta_{1} \delta_{2}}\right) \\
& \quad \times \omega_{\text {mixed }}\left(\mu ; \delta_{1}, \delta_{2}\right) .
\end{aligned}
$$

If we choose $\delta_{1}=\sqrt{\alpha_{r_{1}, p_{1}}}$ and $\delta_{2}=\sqrt{\alpha_{r_{2}, p_{2}}}$, then the desired result is arrived.

\section{Graphics and Error Estimation Tables}

In this section, with the help of the Maple software, we give some plots and error estimation tables for the comparison of the convergence behavior of (29) and (62) operators to the certain functions.

Example 1. Let $\mu(x, y)=\cos \left(x y /\left(1+y^{2}\right)\right)$. In Figure 1, for $r_{1}=r_{2}=5$ (red), $r_{1}=r_{2}=15$ (green), $r_{1}=r_{2}=75$ (blue), and by choosing $p_{1}=p_{2}=1$, we illustrate the convergence of (29) operators to $\mu(x, y)=\cos \left(x y /\left(1+y^{2}\right)\right)$ (yellow). Also, in Table 1, we determine the error of approximation (29) operators to $\mu(x, y)=\cos \left(x y /\left(1+y^{2}\right)\right)$ for the certain values of $-1 \leq x, y \leq 1$ and $r_{1}=r_{2}=5,50,500$, respectively. It is clear from Table 1 that as the values of $r_{1}$ and $r_{2}$ increase then the error of approximation (29) operators to $\mu(x, y)=\cos (x y)$ $\left.\left(1+y^{2}\right)\right)$ decreases.

Example 2. Let $\mu(x, y)=\sin ((5 / 2) x y) e^{-y / 3}$. In Figure 2, for $r_{1}=r_{2}=15, p_{1}=p_{2}=2$, we compare the convergence of (29) (red) and the associated GBS operators (62) (blue) to $\mu$ $(x, y)=\sin ((5 / 2) x y) e^{-y / 3}$ (yellow). Also, in Table 2, we evaluate the error of approximation (29) and (62) operators to $\mu(x, y)=\sin ((5 / 2) x y) e^{-y / 3}$ for $r_{1}=r_{2}=300, p_{1}=p_{2}=2$, and the certain points of $-1 \leq x, y \leq 1$. It is obvious that, for $r_{1}=r_{2}=300, p_{1}=p_{2}=2$, the absolute difference between

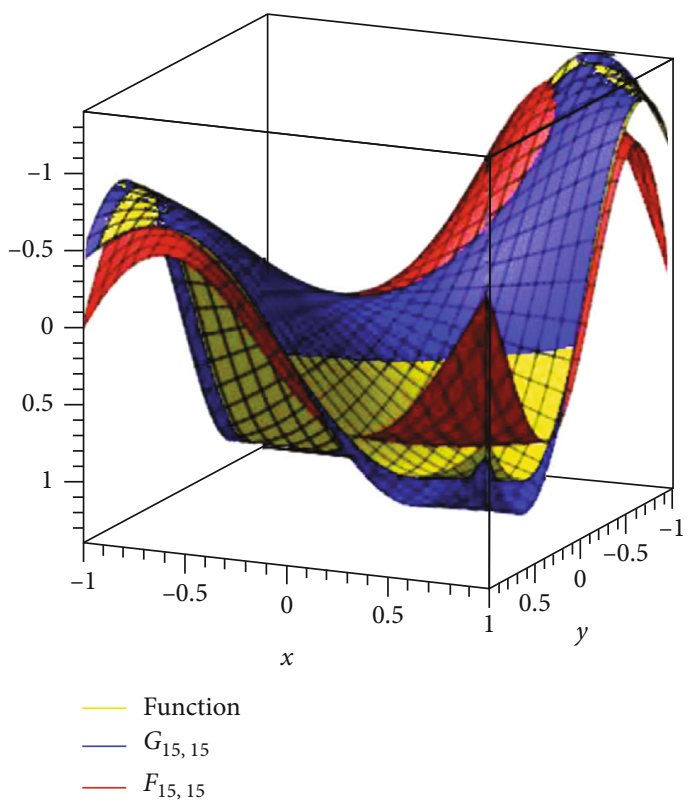

FIGURE 2: The convergence of $F_{r_{1}, r_{2}}(\mu ; x, y)$ (red) and $G_{r_{1}, r_{2}}(\mu ; x, y)$ (blue) operators to $\mu(x, y)=\sin ((5 / 2) x y) e^{-y / 3}$ (yellow) for $r_{1}=r_{2}=$ 15 and $p_{1}=p_{2}=2$.

TABLE 2: Error of approximation $F_{r_{1}, r_{2}}(\mu ; x, y)$ and $G_{r_{1}, r_{2}}(\mu ; x, y)$ operators to $\mu(x, y)=\sin ((5 / 2) x y) e^{-y / 3}$ for $r_{1}=r_{2}=300$ and $p_{1}=$ $p_{2}=2$.

\begin{tabular}{lcc}
\hline$(x, y)$ & $\left|F_{300,300}(\mu ; x, y)-\mu(x, y)\right|$ & $\left|G_{300,300}(\mu ; x, y)-\mu(x, y)\right|$ \\
\hline$(-0.9,0.9)$ & 0.0136597503 & 0.0000249663 \\
$(0.5,0.5)$ & 0.0127076638 & 0.0000753517 \\
$(-0.8,-0.1)$ & 0.0118523844 & 0.0000165295 \\
$(-0.3,-0.6)$ & 0.0117422817 & 0.0000287716 \\
$(-0.6,-0.4)$ & 0.0102228713 & 0.0000248461 \\
$(0.4,0.8)$ & 0.0101988560 & 0.0001669195 \\
$(0.9,0.7)$ & 0.0081455565 & 0.0005454158 \\
$(0.1,-0.4)$ & 0.0070195207 & 0.0000801529 \\
$(-0.7,0.3)$ & 0.0056112239 & 0.0000346506 \\
$(-0.2,0.2)$ & 0.0003416940 & 0.0000879462 \\
\hline
\end{tabular}


(29) operators to $\mu(x, y)$ is greater than that of (62) operators to $\mu(x, y)$. Namely, the (62) operators has better approximation than (29) operators.

\section{Data Availability}

All data required for this paper are included within this paper.

\section{Conflicts of Interest}

The authors declare that there are no conflicts of interest regarding the publication of this paper.

\section{Authors' Contributions}

All the authors contributed equally and significantly in writing this article.

\section{References}

[1] S. Bernstein, "Démonstration du th éorème de Weierstrass fondée sur le calcul des probabilités," Communications de la Société Mathématique de Kharkow, vol. 13, pp. 1-2, 1912.

[2] F. Schurer, Linear Positive Operators in Approximation Theory, Math. Inst. Techn. Univ, Delft Report, 1962.

[3] T. Acar, A. Aral, and V. Gupta, "On approximation properties of a new type of Bernstein-Durrmeyer operators," Mathematica Slovaca, vol. 65, no. 5, pp. 1107-1122, 2015.

[4] A. Izgi, "Approximation by a class of new type Bernstein polynomials of one and two variables," Global Journal of Pure and Applied Mathematics, vol. 8, pp. 55-71, 2012.

[5] X. Chen, J. Tan, Z. Liu, and J. Xie, "Approximation of functions by a new family of generalized Bernstein operators," Journal of Mathematical Analysis and Applications, vol. 450, no. 1, pp. 244-261, 2017.

[6] A. Kajla and T. Acar, "Modified alpha-Bernstein operators with better approximation properties," Annals of Functional Analysis, vol. 10, no. 4, pp. 570-582, 2019.

[7] T. Acar, A. Aral, and S. A. Mohiuddine, "Approximation by bivariate (p, q)-Bernstein-Kantorovich operators," Iranian Journal of Science and Technology, Transactions A: Science, vol. 42, no. 2, pp. 655-662, 2018.

[8] Q. B. Cai, "The Bézier variant of Kantorovich type $\lambda$-Bernstein operators," Journal of Inequalities and Applications, vol. 2018, no. 1, 2018.

[9] T. Acar and A. Kajla, "Degree of approximation for bivariate generalized Bernstein type operators," Results in Mathematics, vol. 73, no. 2, p. 79, 2018.

[10] D. Bărbosu, "Bernstein-Schurer bivariate operators," Revue d'Analyse Numérique et de Théorie de l'Approximation, vol. 33, pp. 157-161, 2004.

[11] L. Căbulea, "Some properties of the Schurer type operators," Acta Universitatis Apulensis. Mathematics-Informatics, vol. 15, pp. 255-261, 2008.

[12] S. Sucu, Y. Done, and E. Ibikli, "Approximation properties of Schurer-Stancu type polynomials," Mathematica Balkanica, vol. 28, 2014.

[13] M. C. Montano and V. Leonessa, "A sequence of Kantorovichtype operators on mobile intervals," Constructive Mathematical Analysis, vol. 2, no. 3, pp. 130-143, 2019.
[14] D. Bărbosu, "The Voronovskaja theorem for BernsteinSchurer operators," Buletinul Științific al Universitatii Baia Mare, Seria B, Fascicola Matematică-Informatică, vol. 18, pp. 137-140, 2002.

[15] A. Kajla and T. Acar, "Blending type approximation by generalized Bernstein-Durrmeyer type operators," Miskolc Mathematical Notes, vol. 19, no. 1, pp. 319-326, 2018.

[16] D. Occorsio, M. G. Russo, and W. Themistoclakis, "Some numerical applications of generalized Bernstein operators," Constructive Mathematical Analysis, vol. 4, no. 2, pp. 186214, 2021.

[17] M. D. Fărcas, "About some bivariate operators of Schurer type," University of Bacău Faculty of Sciences Scientific Studies and Research Series Mathematics and Informatics, vol. 19, no. 1, pp. 73-82, 2009.

[18] M. Mursaleen, M. Nasiruzzaman, and A. Nurgali, "Some approximation results on Bernstein-Schurer operators defined by (p, q)-integers," Journal of Inequalities and Applications, vol. 2015, 12 pages, 2015.

[19] F. Özger, H. M. Srivastava, and S. A. Mohiuddine, "Approximation of functions by a new class of generalized BernsteinSchurer operators," Revista de la Real Academia de Ciencias Exactas, Físicas y Naturales. Serie A. Matemáticas, vol. 114, no. 4, pp. 1-21, 2020.

[20] N. Deo, M. A. Noor, and M. A. Siddiqui, "On approximation by a class of new Bernstein type operators," Applied Mathematics and Computation, vol. 201, no. 1-2, pp. 604-612, 2008.

[21] S. A. Mohiuddine, T. Acar, and A. Alotaibi, "Construction of a new family of Bernstein-Kantorovich operators," Mathematicsl Methods in the Applied Sciences, vol. 40, no. 18, pp. 7749-7759, 2017.

[22] A. Kajla, "Generalized Bernstein-Kantorovich-type operators on a triangle," Mathematical Methods in the Applied Sciences, vol. 42, no. 12, pp. 4365-4377, 2019.

[23] A. Kajla and M. Goyal, "Modified Bernstein-Kantorovich operators for functions of one and two variables," Rendiconti del Circolo Matematico di Palermo Series 2, vol. 67, no. 2, pp. 379-395, 2018.

[24] P. P. Korovkin, "On convergence of linear positive operators in the space of continuous functions," Doklady Akademii Nauk SSSR, vol. 90, pp. 961-964, 1953.

[25] F. Altomare and M. Campiti, Korovkin-Type Approximation Theory and Its Applications, vol. 17, Walter de Gruyter, 2011.

[26] V. I. Volkov, "On the convergence of sequences of linear positive operators in the space of continuous functions of two variables," Doklady Akademii Nauk SSSR, vol. 115, pp. 17-19, 1957.

[27] K. Bögel, "Über Mehrdimensionale differentiation von funktionen mehrerer reeller veränderlichen," Journal für die Reine und Angewandte Mathematik, vol. 170, pp. 197-217, 1934.

[28] K. Bögel, "Über mehrdimensionale differentiation, integration und beschränkte variation," Journal für die Reine und Angewandte Mathematik, vol. 173, pp. 5-30, 1935.

[29] E. Dobrescu and I. Matei, "The approximation by Bernstein type polynomials of bidimensional continuous functions," Analele Universitații din Timişoara. Seria Ştiințe MatematiceFizice, vol. 4, pp. 85-90, 1966.

[30] C. Badea, I. Badea, and H. H. Gonska, "Notes on degree of approximation on b-continuous and b-differentiable functions," Approximation Theory and its Applications, vol. 4, pp. 95-108, 1988. 
[31] C. Badea, " $K$-functionals and moduli of smoothness of functions defined on compact metric spaces," Computers \& Mathematics with Applications, vol. 30, no. 3-6, pp. 23-31, 1995.

[32] O. T. Pop and M. D. Fărcas, "Approximation of B-continuous and B-differentiable functions by GBS operators of Bernstein bivariate polynomials," Journal of Inequalities in Pure and Applied Mathematics, vol. 7, p. 9, 2006.

[33] N. İspir, "Quantitative estimates for GBS operators of Chlodowsky-Szász type," Univerzitet u Nišu, vol. 31, pp. 1175-1184, 2017.

[34] A. Kajla and D. Miclăuş, "Blending type approximation by GBS operators of generalized Bernstein-Durrmeyer type," Results in Mathematics, vol. 73, no. 1, p. 1, 2018.

[35] M. Sidharth, N. Ispir, and P. N. Agrawal, "GBS operators of Bernstein-Schurer-Kantorovich type based on q-integers," Applied Mathematics and Computation, vol. 269, pp. 558568, 2015.

[36] T. Acar, A. Aral, and I. Raşa, "Iterated Boolean sums of Bernstein type operators," Numerical Functional Analysis and Optimization, vol. 41, no. 12, pp. 1515-1527, 2020.

[37] M. Mursaleen, M. Ahasan, and K. J. Ansari, "Bivariate Bernstein-Schurer-Stancu type GBS operators in $(p, q)$-analogue," Advances in Difference Equations, vol. 2020, no. 76, 2020.

[38] M. Sidharth, N. Ispir, and P. N. Agrawal, "Approximation of B -continuous and B-differentiable functions by GBS operators of q-Bernstein-Schurer-Stancu type," Turkish Journal of Mathematics, vol. 40, pp. 1298-1315, 2016.

[39] A. M. Acu, T. Acar, C.-V. Muraru, and V. A. Radu, "Some approximation properties by a class of bivariate operators," Mathematical Methods in the Applied Sciences, vol. 42, no. 16, pp. 5551-5565, 2019.

[40] T. Garg, A. M. Acu, and P. N. Agrawal, "Weighted approximation and GBS of Chlodowsky-Szász-Kantorovich type operators," Analysis and Mathematical Physics, vol. 9, no. 3, pp. 1429-1448, 2019.

[41] D. Bărbosu and C. V. Muraru, "Approximating B-continuous functions using GBS operators of Bernstein- Schurer-Stancu type based on $q$-integers," Applied Mathematics and Computation, vol. 259, pp. 80-87, 2015.

[42] S. A. Mohiuddine, "Approximation by bivariate generalized Bernstein-Schurer operators and associated GBS operators," Advances in Difference Equations, vol. 2020, 17 pages, 2020.

[43] K. Bögel, Über die mehrdimensionale Differentiation, vol. 65, Jahresbericht der Deutschen Mathematiker-Vereinigung, 1962. 